\title{
Gravitational form factors of hyperons in light-cone QCD
}

\author{
U. Özdem ${ }^{1,3, *}$ and K. Azizi $\oplus^{2,3, \dagger}$ \\ ${ }^{1}$ Health Services Vocational School of Higher Education, Istanbul Aydin University, \\ Sefakoy-Kucukcekmece, 34295 Istanbul, Turkey \\ ${ }^{2}$ Department of Physics, University of Tehran, North Karegar Avenue, Tehran 14395-547, Iran \\ ${ }^{3}$ Department of Physics, Dogus University, Acibadem-Kadikoy, 34722 Istanbul, Turkey
}

(Received 30 April 2020; accepted 15 June 2020; published 25 June 2020)

\begin{abstract}
The quark parts of the gravitational form factors of hyperons are calculated by means of the light-cone QCD sum rule. In the calculations, the distribution amplitudes (DAs) of $\Sigma, \Xi$, and $\Lambda$ together with the general forms of their interpolating currents as well as the quark part of the energy-momentum tensor current are used. These form factors can provide information on their mass and distributions of the angular momentum, energy and pressure inside the hyperons. It is obtained that the $Q^{2}$ dependencies of the hyperon gravitational form factors are nicely characterized by a multipole fit function. Using the fits of these form factors, some mechanical properties such as the mechanical radius of the hyperons and the pressure and energy distributions at the center of these particles are obtained. The obtained results can help us in better understanding of the internal structures of these baryons and the QCD as theory of the strong interaction.
\end{abstract}

DOI: $10.1103 /$ PhysRevD.101.114026

\section{MOTIVATION}

The gravitational form factors of baryons are fundamental objects that provide valuable information on different observables related to the structure and mechanical properties of these particles. They are described by the help of the matrix element of the energy-momentum tensor [1]. The matrix element of the quark part of the energymomentum tensor current between two hyperonic states is defined as [2],

$$
\begin{aligned}
\left\langle H\left(p^{\prime}, s^{\prime}\right)\left|T_{\mu \nu}^{q}\right| H(p, s)\right\rangle & \\
= & \bar{u}_{H}\left(p^{\prime}, s^{\prime}\right)\left[A^{H-H}\left(Q^{2}\right) \frac{\tilde{P}_{\mu} \tilde{P}_{\nu}}{m_{H}}+i J^{H-H}\left(Q^{2}\right)\right. \\
& \times \frac{\left(\tilde{P}_{\mu} \sigma_{\nu \rho}+\tilde{P}_{\nu} \sigma_{\mu \rho}\right) \Delta^{\rho}}{2 m_{H}}+D^{H-H}\left(Q^{2}\right) \frac{\Delta_{\mu} \Delta_{\nu}-g_{\mu \nu} \Delta^{2}}{4 m_{H}} \\
& \left.+\bar{c}^{H-H}\left(Q^{2}\right) m_{H} g_{\mu \nu}\right] u_{H}(p, s),
\end{aligned}
$$

for $\Sigma-\Sigma, \Xi-\Xi$ and $\Lambda-\Lambda$ transitions. The matrix element for $\Sigma^{0}-\Lambda$ transition can be obtained by the replacement

\footnotetext{
*ulasozdem@aydin.edu.tr

†kazem.azizi@ut.ac.ir
}

Published by the American Physical Society under the terms of the Creative Commons Attribution 4.0 International license. Further distribution of this work must maintain attribution to the author(s) and the published article's title, journal citation, and DOI. Funded by SCOAP ${ }^{3}$.
$m_{H} \rightarrow\left(m_{\Sigma^{0}}+m_{\Lambda}\right) / 2$. In the above equation, $A^{H-H}\left(Q^{2}\right)$, $J^{H-H}\left(Q^{2}\right), D^{H-H}\left(Q^{2}\right)$ and $\bar{c}^{H-H}\left(Q^{2}\right)$ are gravitational form factors, $\tilde{P}=\left(p^{\prime}+p\right) / 2, \Delta=p^{\prime}-p, q=p-p^{\prime}$, $\sigma_{\mu \nu}=\frac{i}{2}\left[\gamma_{\mu}, \gamma_{\nu}\right]$ and $Q^{2}=-\Delta^{2}$. By calculation of the gravitational form factors one can calculate the pressure, shear forces, total angular momentum, and spatial distribution of energy inside the particles [3]. Calculation of gravitational form factors of hadrons is a relatively new subject although they were introduced by Kobzarev and Okun in 1962 [4]. A number of contributions have been made to the calculation of these form factors, recently. Thus, the gravitational form factors of the nucleon have been extracted within various theoretical approaches such as, chiral perturbation theory $(\chi \mathrm{PT})[5-10]$, Bag model [11], instanton picture (IP) [12], chiral quark soliton model $(\chi \mathrm{QSM})$ [13-23], dispersion relation (DR) [24], Skyrme model [25,26], lattice QCD [27-34], light-cone QCD sum rules (LCSR) [35,36], and instant-front form (IFF) [37]. Recently, as a first calculation, the transition gravitational form factors between $N^{*}$ and $N$ baryons have been calculated in the framework of light-cone QCD sum rule [38].

The hyperon states are very interesting as they can be used to check the order of SU(3) flavor symmetry breaking at different interactions. Our knowledge on these particles is very limited because of unstable nature of these particles. This makes experimental studies very difficult and this situation increase the importance of theoretical studies on different aspects of hyperons. In this accordance, we investigate the gravitational form factors defining the 
$\Sigma-\Sigma, \Xi-\Xi, \Lambda-\Lambda$ and the $\Sigma^{0}-\Lambda$ transitions within the light-cone QCD sum rule approach [39-41]. To our best knowledge, this is the first study on the gravitational form factors of the light octet hyperons in the literature. However, there have been attempts to extract the octet and decuplet hyperon's electromagnetic form factors [42-52], axial form factors [45,53-61] as well as their tensor form factors $[62,63]$.

The outline of the paper is as follows: In Sec. II, using LCSR, analytical results are obtained for hyperon's gravitational form factors. Sec. III includes the numerical analyses and Sec. IV is devoted to summary and concluding remarks.

\section{HYPERON'S GRAVITATIONAL FORM FACTORS}

To derive the light-cone QCD sum rules for gravitational form factors of hyperons, we introduce the correlation function

$\Pi_{\mu \nu}(p, q)=i \int d^{4} x e^{i q x}\left\langle 0\left|\mathcal{T}\left[J_{H}(0) T_{\mu \nu}^{q}(x)\right]\right| H(p)\right\rangle$,

where $T_{\mu \nu}^{q}(x)$ is the quark part of the symmetric energymomentum tensor current and $J_{H}(0)$ are interpolating currents for hyperon states. The explicit forms of the $J_{H}(0)$ for different members and $T_{\mu \nu}^{q}(x)$ are given as

$$
\begin{aligned}
& J_{\Sigma}(0)=2 \epsilon^{a b c} \sum_{\ell=1}^{2}\left(u^{a T}(0) C J_{1}^{\ell} s^{b}(0)\right) J_{2}^{\ell} u^{c}(0), \\
& J_{\Xi}(0)=J_{\Sigma}(0)(u \leftrightarrow s), \\
& J_{\Lambda}(0)=\frac{2}{\sqrt{6}} \epsilon^{a b c} \sum_{\ell=1}^{2}\left[2\left(u^{a T}(0) C J_{1}^{\ell} d^{b}(0)\right) J_{2}^{\ell} s^{c}(0)+\left(u^{a T}(0) C J_{1}^{\ell} s^{b}(0)\right) J_{2}^{\ell} d^{c}(0)-\left(d^{a T}(0) C J_{1}^{\ell} s^{b}(0)\right) J_{2}^{\ell} u^{c}(0)\right], \\
& J_{\Sigma^{0}}(0)=\sqrt{2} \epsilon^{a b c} \sum_{\ell=1}^{2}\left[\left(u^{a T}(0) C J_{1}^{\ell} s^{b}(0)\right) J_{2}^{\ell} d^{c}(0)+\left(d^{a T}(0) C J_{1}^{\ell} s^{b}(0)\right) J_{2}^{\ell} u^{c}(0)\right],
\end{aligned}
$$

and

$$
T_{\mu \nu}^{q}(x)=\frac{i}{2}\left[\bar{u}^{d}(x) \stackrel{\leftrightarrow}{D}_{\mu}(x) \gamma_{\nu} u^{d}(x)+\bar{d}^{e}(x) \stackrel{\leftrightarrow}{D}_{\mu}(x) \gamma_{\nu} d^{e}(x)+(\mu \leftrightarrow \nu)\right]
$$

where $J_{1}^{1}=I, J_{1}^{2}=J_{2}^{1}=\gamma_{5}, J_{2}^{2}=t, \stackrel{\leftrightarrow}{D}_{\mu}(x)$ is the two-sided covariant derivative, $C$ is charge conjugation operator, $t$ is arbitrary mixing parameter; and $a, b, c, d$, and $e$ are color indexes. In the above equations, by $\Sigma$ we mean $\Sigma^{+}$.

To obtain the gravitational form factors in light-cone QCD sum rule, we need to calculate the correlation function in two different frameworks. First, we calculate the correlation function in terms of hadronic parameters, known as hadronic side. Second, we obtain the correlation function in terms of the quark-gluon parameters via distribution amplitudes of the related hadron, known as QCD side. In order to suppress undesired contributions, which are related to the excited and continuum states, the Borel transformations are applied. Then, we match both representations of the correlation function by the help of the quark-hadron duality ansatz.

To calculate the correlation function in terms of hadronic properties, a complete hadronic set with the same quantum numbers as the interpolation currents is inserted. Thus, the correlation function takes the form

$$
\Pi_{\mu \nu}^{H a d}(p, q)=\frac{\left\langle 0\left|J_{H}(0)\right| H\left(p^{\prime}, s^{\prime}\right)\right\rangle\left\langle H\left(p^{\prime}, s^{\prime}\right)\left|T_{\mu \nu}^{q}(x)\right| H(p, s)\right\rangle}{m_{H}^{2}-p^{\prime 2}}+\cdots,
$$

where

$$
\left\langle 0\left|J_{H}(0)\right| H\left(p^{\prime}, s^{\prime}\right)\right\rangle=\lambda_{H} u_{H}\left(p^{\prime}, s^{\prime}\right),
$$

with $\lambda_{H}$ and $u_{H}\left(p^{\prime}, s^{\prime}\right)$ being the residue and Dirac spinor corresponding to the hyperon $H$, respectively. Substituting Eqs. (1) and (6) into Eq. (5), we acquire the hadronic representation of the correlation function in terms of the hadronic observables as 


$$
\begin{aligned}
\Pi_{\mu \nu}^{H a d}(p, q)= & \frac{\lambda_{H}}{m_{H}^{2}-p^{\prime 2}}\left(\not \not{ }^{\prime}+m_{H}\right)\left[A^{H-H}\left(Q^{2}\right) \frac{\tilde{P}_{\mu} \tilde{P}_{\nu}}{m_{H}}+i J^{H-H}\left(Q^{2}\right) \frac{\left(\tilde{P}_{\mu} \sigma_{\nu \rho}+\tilde{P}_{\nu} \sigma_{\mu \rho}\right) \Delta^{\rho}}{2 m_{H}}\right. \\
& \left.+D^{H-H}\left(Q^{2}\right) \frac{\Delta_{\mu} \Delta_{\nu}-g_{\mu \nu} \Delta^{2}}{4 m_{H}}+\bar{c}^{H-H}\left(Q^{2}\right) m_{H} g_{\mu \nu}\right] u_{H}(p, s) .
\end{aligned}
$$

The next step is computation of the correlation function in terms of the QCD degrees of freedom as well as the hyperon's distribution amplitudes. Inserting the explicit expressions for the hyperon interpolating currents as well as the quark part of the energy-momentum current into Eq. (3), and performing all contractions among the quark fields with the help of Wick theorem, for the correlation function in $\mathrm{x}$-space, we obtain

$$
\begin{aligned}
& \Pi_{\mu \nu}^{\mathrm{QCD}}(p, q)=-\int d^{4} x e^{i q x}\left[\left\{\left(\gamma_{5}\right)_{\gamma \delta} C_{\alpha \beta}\left(\overleftrightarrow{D}_{\mu}(x) \gamma_{\nu}\right)_{\omega \rho}+t(I)_{\gamma \delta}\left(C \gamma_{5}\right)_{\alpha \beta}\left(\overleftrightarrow{D}_{\mu}(x) \gamma_{\nu}\right)_{\omega \rho}\right.\right. \\
& \left.+\left(\gamma_{5}\right)_{\gamma \delta} C_{\alpha \beta}\left(\stackrel{\leftrightarrow}{D}_{\mu}(x) \gamma_{\mu}\right)_{\omega \rho}+t(I)_{\gamma \delta}\left(C \gamma_{5}\right)_{\alpha \beta}\left(\stackrel{\leftrightarrow}{D}_{\nu}(x) \gamma_{\mu}\right)_{\omega \rho}\right\} \\
& \left.\times\left(\delta_{\sigma}^{\alpha} \delta_{\theta}^{\rho} \delta_{\phi}^{\beta} S_{u}(-x)_{\delta \omega}+\delta_{\sigma}^{\delta} \delta_{\theta}^{\rho} \delta_{\phi}^{\beta} S_{u}(-x)_{\alpha \omega}\right)\left\langle 0\left|\epsilon^{a b c} u_{\sigma}^{a}(0) u_{\theta}^{b}(x) s_{\phi}^{c}(0)\right| \Sigma(p)\right\rangle\right],
\end{aligned}
$$

for $\Sigma-\Sigma$ transition,

$$
\begin{aligned}
\Pi_{\mu \nu}^{\mathrm{QCD}}(p, q)= & -\int d^{4} x e^{i q x}\left[\left\{\left(\gamma_{5}\right)_{\gamma \delta} C_{\alpha \beta}\left(\stackrel{\leftrightarrow}{D}_{\mu}(x) \gamma_{\nu}\right)_{\omega \rho}+t(I)_{\gamma \delta}\left(C \gamma_{5}\right)_{\alpha \beta}\left(\stackrel{\leftrightarrow}{D}_{\mu}(x) \gamma_{\nu}\right)_{\omega \rho}\right.\right. \\
& \left.+\left(\gamma_{5}\right)_{\gamma \delta} C_{\alpha \beta}\left(\stackrel{\leftrightarrow}{D}_{\nu}(x) \gamma_{\mu}\right)_{\omega \rho}+t(I)_{\gamma \delta}\left(C \gamma_{5}\right)_{\alpha \beta}\left(\stackrel{\leftrightarrow}{D}_{\nu}(x) \gamma_{\mu}\right)_{\omega \rho}\right\} \\
& \left.\times \delta_{\sigma}^{\alpha} \delta_{\theta}^{\delta} \delta_{\phi}^{\rho} S_{u}(-x)_{\beta \omega}\left\langle 0\left|\epsilon^{a b c} s_{\sigma}^{a}(0) s_{\theta}^{b}(0) u_{\phi}^{c}(x)\right| \Xi(p)\right\rangle\right]
\end{aligned}
$$

for $\Xi-\Xi$ transition,

$$
\begin{aligned}
\Pi_{\mu \nu}^{\mathrm{QCD}}(p, q)= & -\frac{1}{\sqrt{6}} \int d^{4} x e^{i q x}\left[\left\{\left(\gamma_{5}\right)_{\gamma \delta} C_{\alpha \beta}\left(\stackrel{\leftrightarrow}{D}_{\mu}(x) \gamma_{\nu}\right)_{\omega \rho}+t(I)_{\gamma \delta}\left(C \gamma_{5}\right)_{\alpha \beta}\left(\stackrel{\leftrightarrow}{D}_{\mu}(x) \gamma_{\nu}\right)_{\omega \rho}\right.\right. \\
& \left.+\left(\gamma_{5}\right)_{\gamma \delta} C_{\alpha \beta}\left(\stackrel{\leftrightarrow}{D}_{\nu}(x) \gamma_{\mu}\right)_{\omega \rho}+t(I)_{\gamma \delta}\left(C \gamma_{5}\right)_{\alpha \beta}\left(\stackrel{\leftrightarrow}{D}_{\nu}(x) \gamma_{\mu}\right)_{\omega \rho}\right\} \\
& \times\left\{\left(2 \delta_{\sigma}^{\rho} \delta_{\theta}^{\beta} \delta_{\phi}^{\delta} S_{u}(-x)_{\alpha \omega}+\delta_{\sigma}^{\rho} \delta_{\theta}^{\delta} \delta_{\phi}^{\beta} S_{u}(-x)_{\alpha \omega}-\delta_{\sigma}^{\rho} \delta_{\theta}^{\alpha} \delta_{\phi}^{\beta} S_{u}(-x)_{\delta \omega}\right)\left\langle 0\left|\epsilon^{a b c} u_{\sigma}^{a}(x) d_{\theta}^{b}(0) s_{\phi}^{c}(0)\right| \Lambda(p)\right\rangle\right. \\
& \left.\left.+\left(2 \delta_{\sigma}^{\beta} \delta_{\theta}^{\rho} \delta_{\phi}^{\delta} S_{d}(-x)_{\beta \omega}+\delta_{\sigma}^{\alpha} \delta_{\theta}^{\rho} \delta_{\phi}^{\beta} S_{d}(-x)_{\delta \omega}-\delta_{\sigma}^{\delta} \delta_{\theta}^{\rho} \delta_{\phi}^{\beta} S_{d}(-x)_{\alpha \omega}\right)\left\langle 0\left|\epsilon^{a b c} u_{\sigma}^{a}(0) d_{\theta}^{b}(x) s_{\phi}^{c}(0)\right| \Lambda(p)\right\rangle\right\}\right],
\end{aligned}
$$

for $\Lambda-\Lambda$ transition and

$$
\begin{aligned}
\Pi_{\mu \nu}^{\mathrm{QCD}}(p, q)= & -\frac{\sqrt{2}}{2} \int d^{4} x e^{i q x}\left[\left\{\left(\gamma_{5}\right)_{\gamma \delta} C_{\alpha \beta}\left(\stackrel{\leftrightarrow}{D}_{\mu}(x) \gamma_{\nu}\right)_{\omega \rho}+t(I)_{\gamma \delta}\left(C \gamma_{5}\right)_{\alpha \beta}\left(\stackrel{\leftrightarrow}{D}_{\mu}(x) \gamma_{\nu}\right)_{\omega \rho}\right.\right. \\
& \left.+\left(\gamma_{5}\right)_{\gamma \delta} C_{\alpha \beta}\left(\stackrel{\leftrightarrow}{D}_{\nu}(x) \gamma_{\mu}\right)_{\omega \rho}+t(I)_{\gamma \delta}\left(C \gamma_{5}\right)_{\alpha \beta}\left(\stackrel{\leftrightarrow}{D}_{\nu}(x) \gamma_{\mu}\right)_{\omega \rho}\right\} \\
& \times\left\{\left(\delta_{\sigma}^{\rho} \delta_{\theta}^{\delta} \delta_{\phi}^{\beta} S_{u}(-x)_{\alpha \omega}+\delta_{\sigma}^{\rho} \delta_{\theta}^{\alpha} \delta_{\phi}^{\beta} S_{u}(-x)_{\delta \omega}\right)\left\langle 0\left|\epsilon^{a b c} u_{\sigma}^{a}(x) d_{\theta}^{b}(0) s_{\phi}^{c}(0)\right| \Lambda(p)\right\rangle\right. \\
& \left.\left.+\left(\delta_{\sigma}^{\alpha} \delta_{\theta}^{\rho} \delta_{\phi}^{\beta} S_{d}(-x)_{\delta \omega}+\delta_{\sigma}^{\delta} \delta_{\theta}^{\rho} \delta_{\phi}^{\beta} S_{d}(-x)_{\alpha \omega}\right)\left\langle 0\left|\epsilon^{a b c} u_{\sigma}^{a}(0) d_{\theta}^{b}(x) s_{\phi}^{c}(0)\right| \Lambda(p)\right\rangle\right\}\right],
\end{aligned}
$$

for $\Sigma^{0}-\Lambda$ transition. Here $S_{q}(x)$ is the light-quark propagator and it is given as

$$
S_{q}(x)=\frac{1}{2 \pi^{2} x^{2}}\left(i \frac{\not x}{x^{2}}-\frac{m_{q}}{2}\right)-\frac{\langle\bar{q} q\rangle}{12}\left(1-i \frac{m_{q} \not \hat{x}}{4}\right)-\frac{\langle\bar{q} \sigma \cdot G q\rangle}{192} x^{2}\left(1-i \frac{m_{q} \not x}{6}\right)-\frac{i g_{s}}{32 \pi^{2} x^{2}} G^{\mu \nu}(x)\left[\not \sigma_{\mu \nu}+\sigma_{\mu \nu} x\right] .
$$

The $\left\langle 0\left|\epsilon^{a b c} u_{\sigma}^{a}\left(a_{1} x\right) u_{\theta}^{b}\left(a_{2} x\right) d_{\phi}^{c}\left(a_{3} x\right)\right| \Sigma(p)\right\rangle, \quad\left\langle 0\left|\epsilon^{a b c} s_{\sigma}^{a}\left(a_{1} x\right) s_{\theta}^{b}\left(a_{2} x\right) u_{\phi}^{c}\left(a_{3} x\right)\right| \Xi(p)\right\rangle \quad$ and $\quad\langle 0| \epsilon^{a b c} u_{\sigma}^{a}\left(a_{1} x\right) d_{\theta}^{b}\left(a_{2} x\right) s_{\phi}^{c} \times$ $\left(a_{3} x\right)|\Lambda(p)\rangle$ matrix elements are the expressions including the distribution amplitudes of the hyperon states and they are necessary for further evaluations. The explicit form of this matrix elements in terms of DAs together with the explicit forms of DAs for hyperons are presented in the Refs. [64,65]. The shape parameters of DAs of hyperons are calculated in the framework of lattice QCD [66], baryon chiral perturbation theory [67] and QCD sum rules [64,65]. After using the explicit forms of the above matrix elements and the light-quark propagator, we obtain an expressions in $\mathrm{x}$-space. The next step is to 
perform the Fourier integrals. These procedures are standard in the method used but very lengthy. Thus, we do not present these steps here.

The desired sum rules are acquired by matching both representations of the correlation function. To do this, different and independent Lorentz structures are needed to be chosen. For this purpose, we choose $p_{\mu} q_{\nu}, p_{\mu} \gamma_{\nu} \phi, q_{\mu} q_{\nu}, g_{\mu \nu}$ structures for $A^{H-H}\left(Q^{2}\right), J^{H-H}\left(Q^{2}\right), D^{H-H}\left(Q^{2}\right)$, and $\bar{c}^{H-H}\left(Q^{2}\right)$ form factors, respectively. As a result, we obtain the sum rules,

$$
\begin{aligned}
& \frac{\lambda_{\Sigma}}{m_{\Sigma}^{2}-p^{\prime 2}} A^{\Sigma-\Sigma}\left(Q^{2}\right)=-\Pi_{1}^{\mathrm{QCD}}\left(Q^{2}\right), \quad \frac{\lambda_{\Sigma}}{m_{\Sigma}^{2}-p^{2}} J^{\Sigma-\Sigma}\left(Q^{2}\right)=\Pi_{2}^{\mathrm{QCD}}\left(Q^{2}\right), \\
& \frac{\lambda_{\Sigma}}{m_{\Sigma}^{2}-p^{\prime 2}} D^{\Sigma-\Sigma}\left(Q^{2}\right)=2 \Pi_{3}^{\mathrm{QCD}}\left(Q^{2}\right), \quad \frac{\lambda_{\Sigma}}{m_{\Sigma}^{2}-p^{\prime 2}} \bar{c}^{\Sigma-\Sigma}\left(Q^{2}\right)=\frac{1}{2 m_{\Sigma}^{2}} \Pi_{4}^{\mathrm{QCD}}\left(Q^{2}\right), \\
& \frac{\lambda_{\Xi}}{m_{\Xi}^{2}-p^{\prime 2}} A^{\Xi-\Xi}\left(Q^{2}\right)=-\Pi_{5}^{\mathrm{QCD}}\left(Q^{2}\right), \quad \frac{\lambda_{\Xi}}{m_{\Xi}^{2}-p^{\prime 2}} J^{\Xi-\Xi}\left(Q^{2}\right)=\Pi_{6}^{\mathrm{QCD}}\left(Q^{2}\right), \\
& \frac{\lambda_{\Xi}}{m_{\Xi}^{2}-p^{\prime 2}} D^{\Xi-\Xi}\left(Q^{2}\right)=2 \Pi_{7}^{\mathrm{QCD}}\left(Q^{2}\right), \quad \frac{\lambda_{\Xi}}{m_{\Xi}^{2}-p^{\prime 2}} \bar{c}^{\Xi-\Xi}\left(Q^{2}\right)=\frac{1}{2 m_{\Xi}^{2}} \Pi_{8}^{\mathrm{QCD}}\left(Q^{2}\right), \\
& \frac{\lambda_{\Lambda}}{m_{\Lambda}^{2}-p^{\prime 2}} A^{\Lambda-\Lambda}\left(Q^{2}\right)=-\Pi_{9}^{\mathrm{QCD}}\left(Q^{2}\right), \quad \frac{\lambda_{\Lambda}}{m_{\Lambda}^{2}-p^{\prime 2}} J^{\Lambda-\Lambda}\left(Q^{2}\right)=\Pi_{10}^{\mathrm{QCD}}\left(Q^{2}\right), \\
& \frac{\lambda_{\Lambda}}{m_{\Lambda}^{2}-p^{\prime 2}} D^{\Lambda-\Lambda}\left(Q^{2}\right)=2 \Pi_{11}^{\mathrm{QCD}}\left(Q^{2}\right), \quad \frac{\lambda_{\Lambda}}{m_{\Lambda}^{2}-p^{\prime 2}} \bar{c}^{\Lambda-\Lambda}\left(Q^{2}\right)=\frac{1}{2 m_{\Lambda}^{2}} \Pi_{12}^{\mathrm{QCD}}\left(Q^{2}\right), \\
& \frac{\lambda_{\Sigma^{0}}}{m_{\Sigma^{0}}^{2}-p^{\prime 2}} A^{\Sigma^{0}-\Lambda}\left(Q^{2}\right)=-\Pi_{13}^{\mathrm{QCD}}\left(Q^{2}\right), \quad \frac{\lambda_{\Sigma^{0}}}{m_{\Sigma^{0}}^{2}-p^{\prime 2}} J^{\Sigma^{0}-\Lambda}\left(Q^{2}\right)=\Pi_{14}^{\mathrm{QCD}}\left(Q^{2}\right), \\
& \frac{\lambda_{\Sigma^{0}}}{m_{\Sigma^{0}}^{2}-p^{\prime 2}} D^{\Sigma^{0}-\Lambda}\left(Q^{2}\right)=2 \Pi_{15}^{\mathrm{QCD}}\left(Q^{2}\right), \quad \frac{\lambda_{\Sigma^{0}}}{m_{\Sigma^{0}}^{2}-p^{\prime 2}} \bar{c}^{\Sigma^{0}-\Lambda}\left(Q^{2}\right)=\frac{2}{\left(m_{\Lambda}+m_{\Sigma^{0}}\right)^{2}} \Pi_{16}^{\mathrm{QCD}}\left(Q^{2}\right) .
\end{aligned}
$$

The $\Pi_{i}^{\mathrm{QCD}}\left(Q^{2}\right)$ functions appear in Eqs. (13)-(16) are very lengthy. Hence, as an example, we present the result of the $\Pi_{1}^{\mathrm{QCD}}\left(Q^{2}\right)$ :

$$
\begin{aligned}
\Pi_{1}^{\mathrm{QCD}}\left(Q^{2}\right)= & \frac{m_{\Sigma}}{4} \int_{0}^{1} d x_{2} \int_{0}^{1-x_{2}} d x_{1} \frac{x_{2}}{\left(q-p x_{2}\right)^{2}}\left[(1-t)\left(3 A_{1}+6 A_{3}-V_{1}+2 V_{3}\right)+2(1+t)\left(P_{1}-S_{1}-T_{1}\right.\right. \\
& \left.\left.+2 T_{7}\right)\right]\left(x_{1}, x_{2}, 1-x_{1}-x_{2}\right)+\frac{m_{\Sigma}}{4} \int_{0}^{1} d \alpha \int_{\alpha}^{1} d x_{2} \int_{0}^{1-x_{2}} d x_{1} \frac{1}{(q-p \alpha)^{2}}\left[2 ( 1 - t ) \left(2 A_{1}-2 A_{2}+2 A_{3}\right.\right. \\
& \left.\left.-V_{1}+V_{2}+V_{3}\right)+(1+t)\left(7 T_{1}+4 T_{2}+3 T_{3}+11 T_{7}\right)\right]\left(x_{1}, x_{2}, 1-x_{1}-x_{2}\right) \\
& +\frac{m_{\Sigma}^{3}}{2} \int_{0}^{1} d \alpha \int_{\alpha}^{1} d x_{2} \int_{0}^{1-x_{2}} d x_{1} \frac{\alpha^{2}}{(q-p \alpha)^{4}}\left[(1-t)\left(3 A_{1}-3 A_{2}-A_{4}-A_{5}-V_{1}+V_{2}+2 V_{3}-V_{4}\right)\right. \\
& \left.+(1+t)\left(-P_{1}+P_{2}-S_{1}+S_{2}-2 T_{1}-T_{3}+T_{5}+6 T_{7}\right)\right]\left(x_{1}, x_{2}, 1-x_{1}-x_{2}\right) \\
& +\frac{m_{\Sigma}^{3}}{4} \int_{0}^{1} d \beta \int_{\beta}^{1} d_{\alpha} \int_{\alpha}^{1} d x_{2} \int_{0}^{1-x_{2}} d x_{1} \frac{\beta}{(q-p \beta)^{4}}\left[4 ( 1 - t ) \left(2 A_{1}-2 A_{2}+2 A_{3}+2 A_{4}-2 A_{5}+2 A_{6}\right.\right. \\
& \left.-V_{1}+V_{2}+V_{3}+V_{4}+V_{5}-V_{6}\right)+(1+t)\left(14 T_{1}+9 T_{2}+5 T_{3}+5 T_{4}+9 T_{5}-14 T_{6}+23 T_{7}\right. \\
& \left.\left.+23 T_{8}\right)\right]\left(x_{1}, x_{2}, 1-x_{1}-x_{2}\right)+2 m_{\Sigma}^{5} \int_{0}^{1} d \beta \int_{\beta}^{1} d_{\alpha} \int_{\alpha}^{1} d x_{2} \int_{0}^{1-x_{2}} d x_{1} \\
& \times \frac{\beta^{3}}{(q-p \beta)^{6}}\left[(1+t)\left(T_{2}-T_{3}-T_{4}+T_{5}+T_{7}+T_{8}\right)\right]\left(x_{1}, x_{2}, 1-x_{1}-x_{2}\right) .
\end{aligned}
$$

Here, $S_{i}, P_{i}, A_{i}, V_{i}$, and $T_{i}$ are DAs of different twists. They are expressed in terms of hyperon's wave functions. The wave functions are also functions of different parameters that all are presented in Refs. [64,65]. In order to remove the unwanted contributions corresponding to the excited and continuum states, the Borel transformation and continuum subtraction are applied. Following these procedures, the contributions of the higher states and continuum are exponentially suppressed. The Borel transformation and the continuum subtraction are carried out by employing the subsequent replacement rules [68]: 


$$
\begin{aligned}
& \int d z \frac{\rho(z)}{\Delta^{2}} \rightarrow-\int_{x_{0}}^{1} \frac{d z}{z} \rho(z) e^{-s(z) / M^{2}}, \\
& \int d z \frac{\rho(z)}{\Delta^{4}} \rightarrow \frac{1}{M^{2}} \int_{x_{0}}^{1} \frac{d z}{z^{2}} \rho(z) e^{-s(z) / M^{2}}+\frac{\rho\left(x_{0}\right)}{Q^{2}+x_{0}^{2} m_{H}^{2}} e^{-s_{0} / M^{2}}, \\
& \int d z \frac{\rho(z)}{\Delta^{6}} \rightarrow-\frac{1}{2 M^{4}} \int_{x_{0}}^{1} \frac{d z}{z^{3}} \rho(z) e^{-s(z) / M^{2}}-\frac{1}{2 M^{2}} \frac{\rho\left(x_{0}\right)}{x_{0}\left(Q^{2}+x_{0}^{2} m_{H}^{2}\right)} e^{-s_{0} / M^{2}}+\frac{1}{2} \frac{x_{0}^{2}}{Q^{2}+x_{0}^{2} m_{H}^{2}}\left[\frac{d}{d x_{0}} \frac{\rho\left(x_{0}\right)}{x_{0}\left(Q^{2}+x_{0}^{2} m_{H}^{2}\right)}\right] e^{-s_{0} / M^{2}},
\end{aligned}
$$

where,

$$
\begin{aligned}
\Delta & =q-z p, \\
s(z) & =(1-z) m_{H}^{2}+\frac{1-z}{z} Q^{2}, \\
x_{0} & =\left(\sqrt{\left(Q^{2}+s_{0}-m_{H}^{2}\right)^{2}+4 m_{H}^{2} Q^{2}}-\left(Q^{2}+s_{0}-m_{H}^{2}\right)\right) / 2 m_{H}^{2} .
\end{aligned}
$$

\section{NUMERICAL RESULTS}

This section is dedicated to the numerical analysis for gravitational form factors of hyperon states. The distribution amplitudes of the $\Sigma, \Xi$ and $\Lambda$ baryons have been evaluated in the framework of QCD sum rule in Refs. [64,65]. In Table I, we present the numerical values of the input parameters inside the DAs of hyperons from these studies, which are used in the numerical computations. For the numerical computations, the hyperon masses are also taken as, $m_{\Sigma}=1189.37 \pm 0.07 \mathrm{MeV}, m_{\Xi}=1314.86 \pm 0.20 \mathrm{MeV}$, $m_{\Sigma^{0}}=1192.642 \pm 0.024 \mathrm{MeV}$ and $m_{\Lambda}=1115.685 \pm$ $0.006 \mathrm{MeV}$ [69]. Additionally, we need the values of the residues of $\Sigma, \Xi, \Sigma^{0}$ and $\Lambda$ baryons, which are borrowed from [48].

The estimations for the gravitational form factors of hyperon states depend on several auxiliary parameters: mixing parameter $t$, the Borel mass parameter $M^{2}$ and continuum threshold $s_{0}$. Based on the standard prescriptions of the approach used, the physical observables should weakly depend on these auxiliary parameters. The arbitrary mixing parameter $t$ is chosen such that, the predictions of the gravitational form factors are practically independent of the values of $t$. From the numerical analyzes, it is seen that in the region $-0.19 \leq \cos \theta \leq-0.44$ the gravitational form factors weakly depend on $t$, where $\tan \theta=t$. The working interval for the continuum threshold $s_{0}$ is obtained considering the fact that the gravitational form factors also weakly depend on this parameter. It is not totally arbitrary, but depend on the energy of the first excited states in the channels under consideration. We follow the steps described below to get the working window of Borel mass parameter $M^{2}$. The lower limit of $M^{2}$ is obtained requiring that the perturbative contribution exceeds over the nonperturbative one and the series of nonperturbative operators are convergent. The upper limit of $M^{2}$ is obtained by the requirement that the contributions of continuum and higher states should be less than the ground state contribution.

Performed numerical calculations show that the regions

$$
\begin{array}{ll}
2.30 \mathrm{GeV}^{2} \leq s_{0} \leq 3.00 \mathrm{GeV}^{2} & \text { for } \Sigma-\Sigma, \\
2.90 \mathrm{GeV}^{2} \leq s_{0} \leq 3.60 \mathrm{GeV}^{2} & \text { for } \Xi-\Xi, \\
2.00 \mathrm{GeV}^{2} \leq s_{0} \leq 2.60 \mathrm{GeV}^{2} & \text { for } \Lambda-\Lambda, \\
2.30 \mathrm{GeV}^{2} \leq s_{0} \leq 3.00 \mathrm{GeV}^{2} & \text { for } \Sigma^{0}-\Lambda, \\
2.00 \mathrm{GeV}^{2} \leq M^{2} \leq 3.00 \mathrm{GeV}^{2} & \text { for } \Sigma-\Sigma, \\
2.50 \mathrm{GeV}^{2} \leq M^{2} \leq 3.50 \mathrm{GeV}^{2} & \text { for } \Xi-\Xi, \\
2.00 \mathrm{GeV}^{2} \leq M^{2} \leq 3.00 \mathrm{GeV}^{2} & \text { for } \Lambda-\Lambda, \\
2.00 \mathrm{GeV}^{2} \leq M^{2} \leq 3.00 \mathrm{GeV}^{2} & \text { for } \Sigma^{0}-\Lambda,
\end{array}
$$

TABLE I. The numerical values of the parameters in the wavefunctions of hyperons taken from Refs. $[64,65]$.

\begin{tabular}{lcc}
\hline \hline \multicolumn{3}{c}{ Parameters in wave functions of hyperons } \\
\hline$\Sigma$ & \multicolumn{1}{c}{$\Xi$} & $\Lambda$ \\
\hline$f=(9.4 \pm 0.4) \times 10^{-3} \mathrm{GeV}^{2}$ & $f=(9.9 \pm 0.4) \times 10^{-3} \mathrm{GeV}^{2}$ & $f=(6.0 \pm 0.3) \times 10^{-3} \mathrm{GeV}^{2}$ \\
$\lambda_{1}=(-2.5 \pm 0.1) \times 10^{-2} \mathrm{GeV}^{2}$ & $\lambda_{1}=(-2.8 \pm 0.1) \times 10^{-2} \mathrm{GeV}^{2}$ & $\lambda_{1}=(1.0 \pm 0.3) \times 10^{-2} \mathrm{GeV}^{2}$ \\
$\lambda_{2}=(4.4 \pm 0.1) \times 10^{-2} \mathrm{GeV}^{2}$ & $\lambda_{2}=(5.2 \pm 0.2) 10^{-2} \times \mathrm{GeV}^{2}$ & $\lambda_{2}=(0.83 \pm 0.05) \times 10^{-2} \mathrm{GeV}^{2}$ \\
$\lambda_{3}=(2.0 \pm 0.1) \times 10^{-2} \mathrm{GeV}^{2}$ & $\lambda_{3}=(0.17 \pm 0.1) \times 10^{-2} \mathrm{GeV}^{2}$ & $\lambda_{3}=(0.83 \pm 0.05) \times 10^{-2} \mathrm{GeV}^{2}$ \\
\hline \hline
\end{tabular}




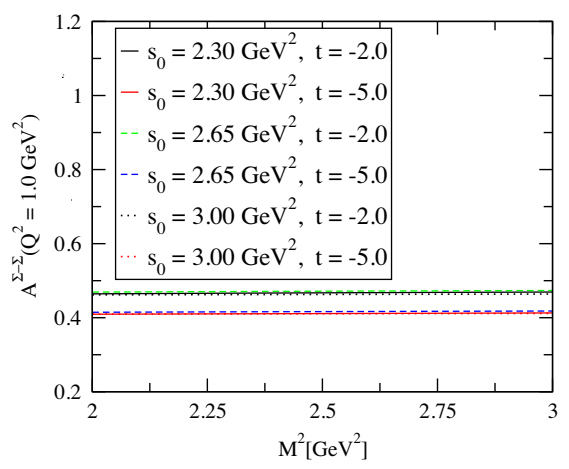

(a)

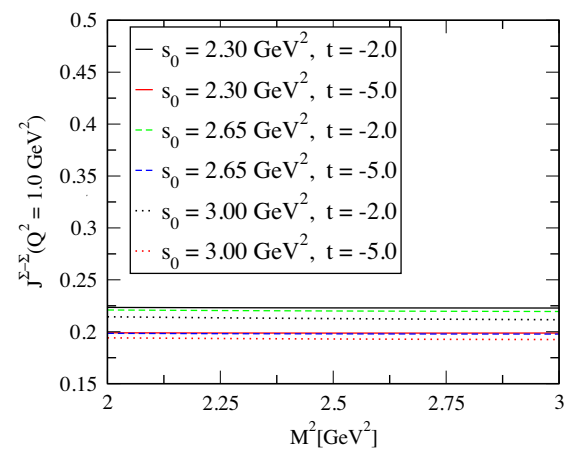

(d)

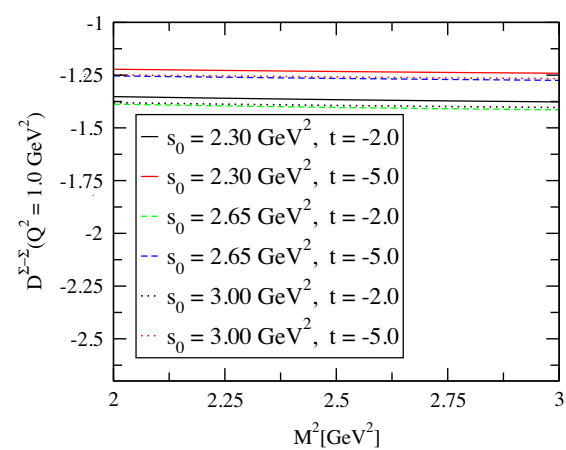

(g)

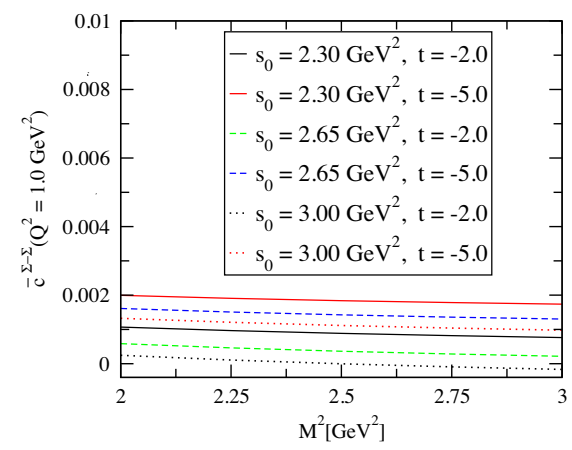

(j)

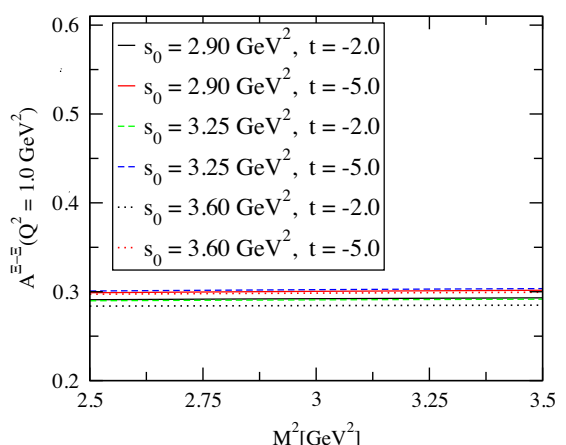

(b)

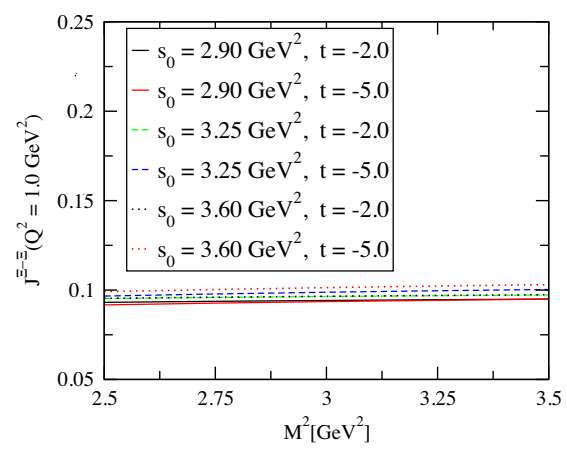

(e)

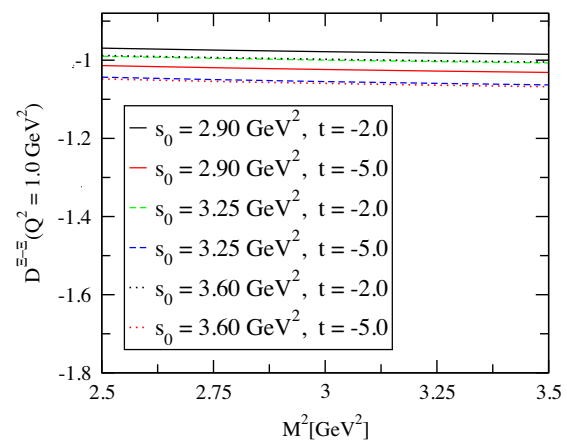

(h)

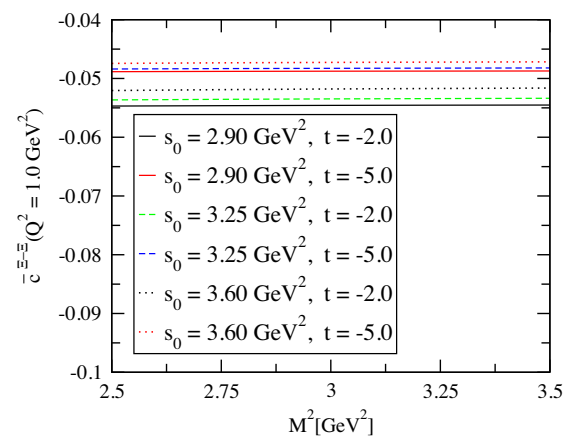

(k)

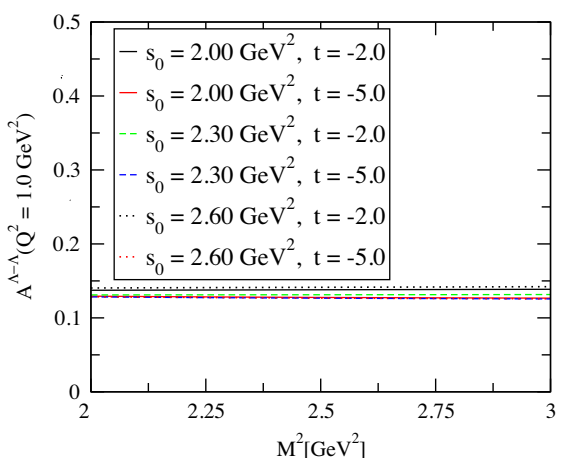

(c)

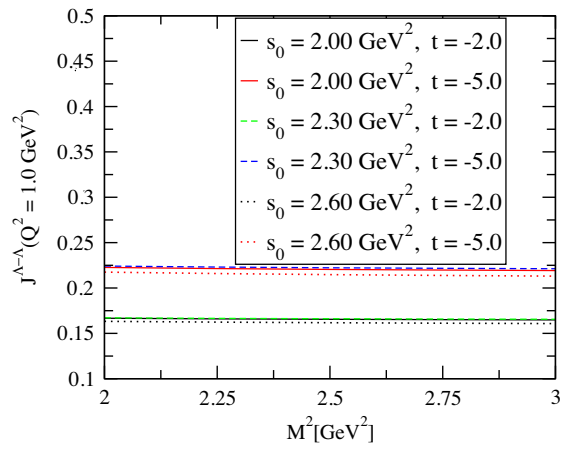

(f)

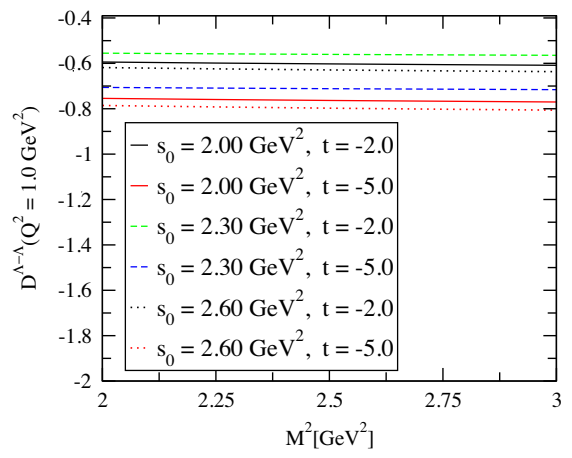

(i)

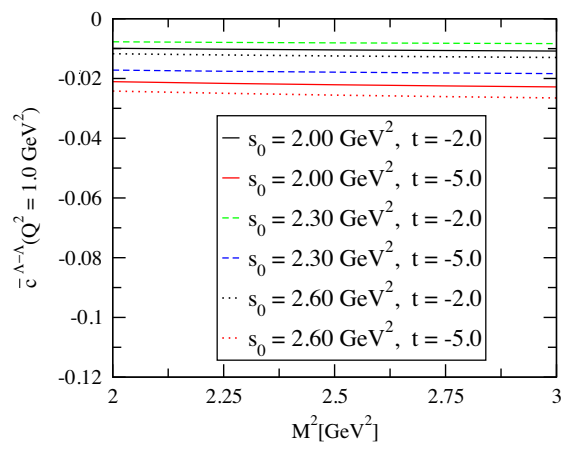

(l)

FIG. 1. The dependence of the gravitational form factors of hyperons on $M^{2}$ at $Q^{2}=1.0 \mathrm{GeV}^{2}$ and different values of $s_{0}$ and $t$ at their working windows. (a), (d), (g) and (j) for $\Sigma$ baryon, (b), (e), (h), and (k) for $\Xi$ baryon and; (c), (f), (i), and (l) for $\Lambda$ baryon. 
satisfy all the aforementioned constraints on $M^{2}$ and $s_{0}$. To visualize effects of $t, M^{2}$, and $s_{0}$ on the gravitational form factors we depict the dependence of these form factors on the auxiliary parameters in Fig. 1. As it can be seen from this figure, the gravitational form factors are quite stable against the variation of these parameters in their working windows. Thus the selected working intervals for the auxiliary parameters satisfy the criteria of the method. In Fig. 2 we present dependence of the gravitational form factors $A^{H-H}\left(Q^{2}\right), J^{H-H}\left(Q^{2}\right), D^{H-H}\left(Q^{2}\right)$, and $\bar{c}^{H-H}\left(Q^{2}\right)$

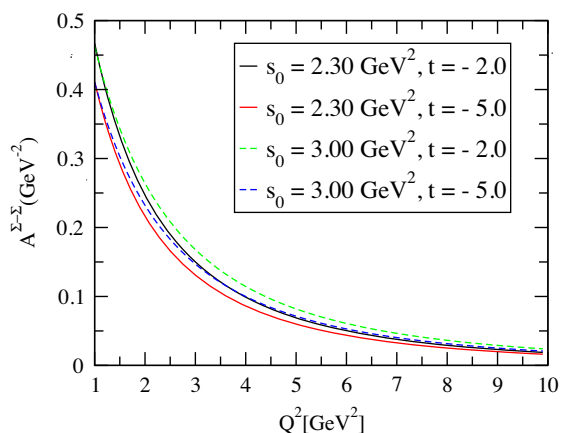

(a)

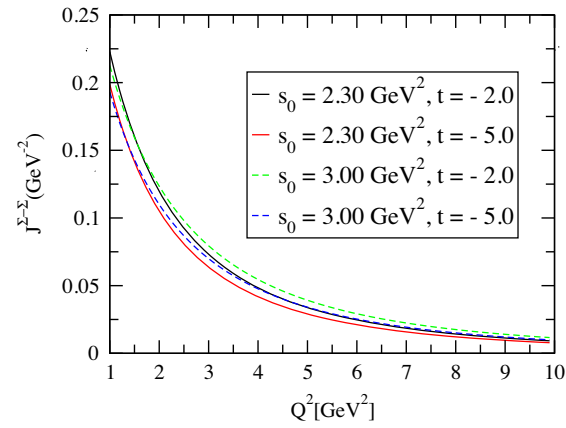

(d)

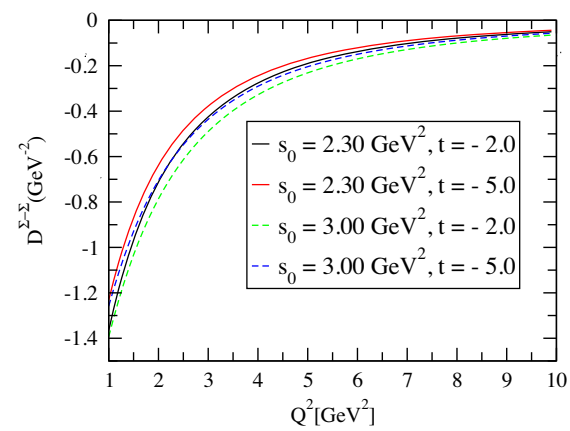

(g)

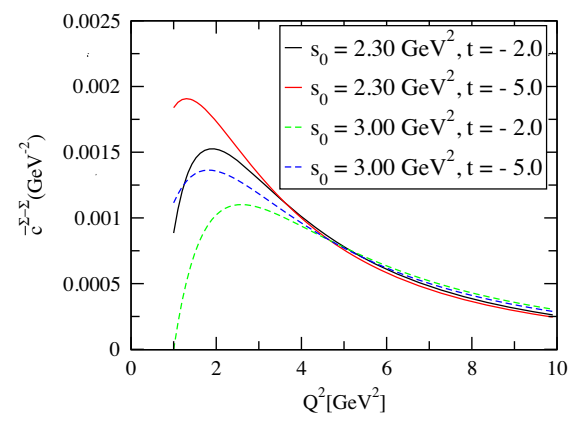

(j)

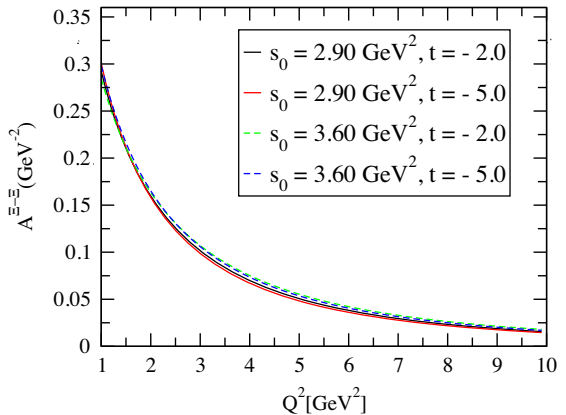

(b)

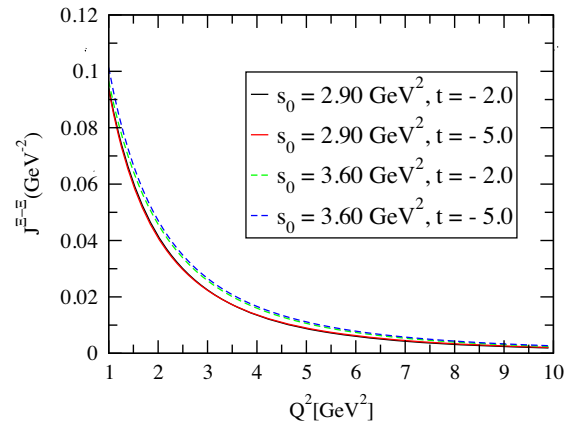

(e)

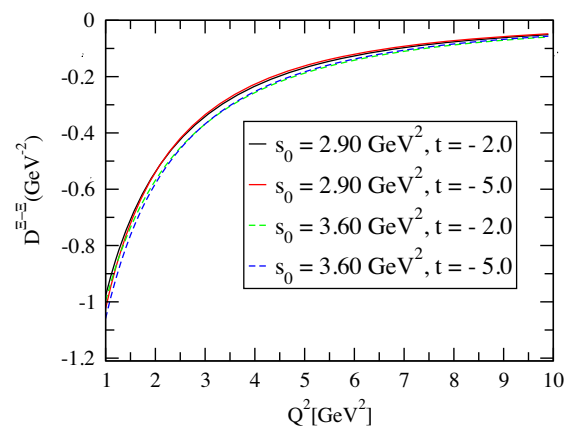

(h)

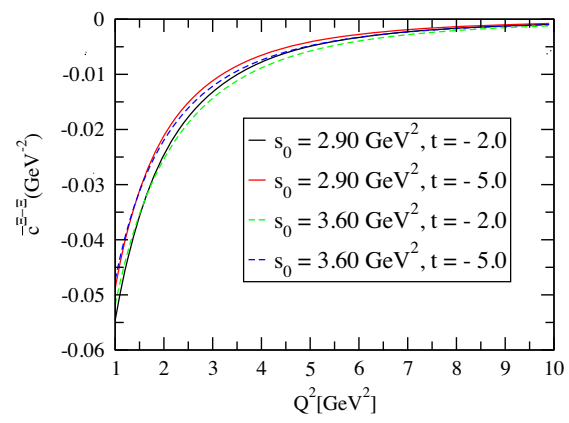

(k)

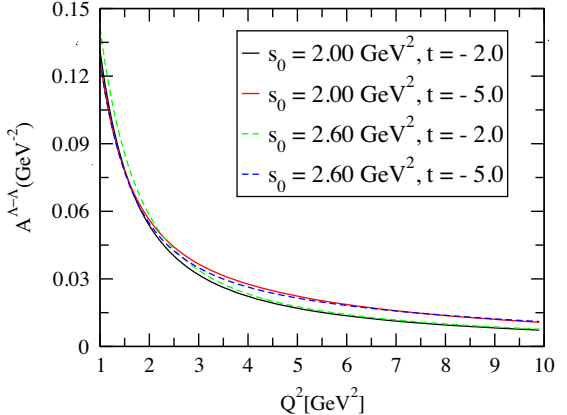

(c)

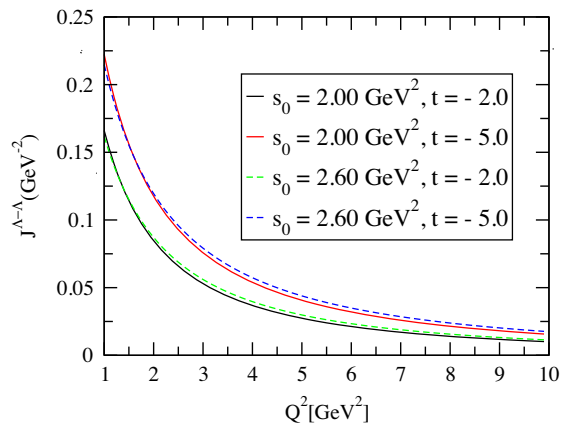

(f)

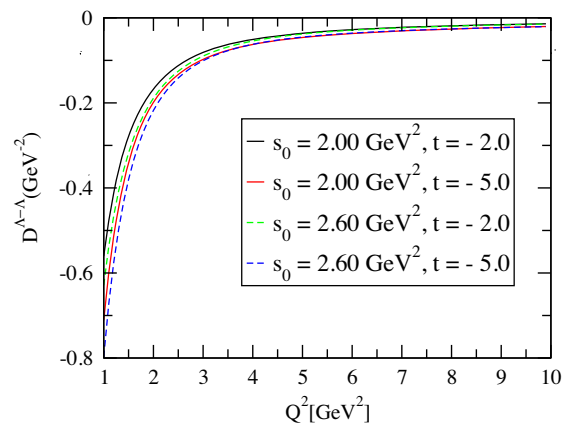

(i)

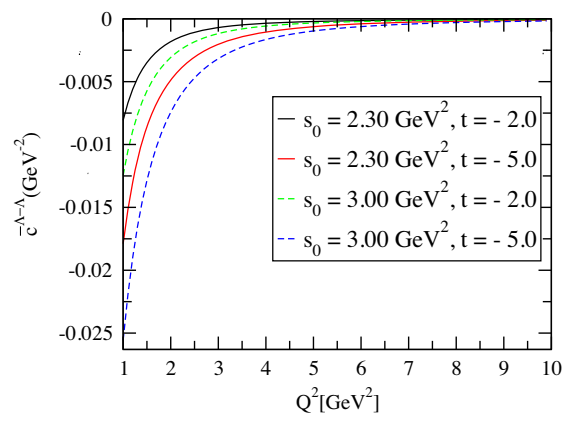

(1)

FIG. 2. The dependence of the gravitational form factors of hyperons on $Q^{2}$ at $M^{2}=2.5 \mathrm{GeV}^{2}$ and different values of $s_{0}$ and $t$ at their working windows. (a), (d), (g) and (j) for $\Sigma$ baryon, (b), (e), (h), and (k) for $\Xi$ baryon and; (c), (f), (i), and (l) for $\Lambda$ baryon. 
on $Q^{2}$ in the interval $1.0 \mathrm{GeV}^{2} \leq Q^{2} \leq 10.0 \mathrm{GeV}^{2}$ at fixed values of auxiliary parameters, using the central values of all input parameters in DAs. From this figure, we see that the $Q^{2}$ dependencies of the gravitational form factors are smoothly varying and decrease with increasing of the $Q^{2}$ as expected.The only exception is the form factor $\bar{c}^{\Sigma-\Sigma}\left(Q^{2}\right)$. The behaviour of this form factor is not reliable. Therefore, we will not present the fit result of this form factor. Our numerical results for the transitional gravitational form factors of $\Sigma^{0}-\Lambda$ transition are very small and close to zero, hence, we do not show the $Q^{2}$ and $M^{2}$ dependence of the form factors corresponding to this transition.

To extrapolate the gravitational form factors to lowmomentum region, $Q^{2}=0$, we fit the light-cone QCD sum rule results to different forms of monopole and dipole, which unluckily fail to give reasonable characterizations of data with a two-parameter fit. However, our numerical studies indicate that the gravitational form factors of hyperons are nicely characterized employing the multipole fit function,

$$
F^{H-H}\left(Q^{2}\right)=\frac{F^{H-H}(0)}{\left(1+Q^{2} m_{p}\right)^{p}},
$$

where $F^{H-H}\left(Q^{2}\right)=A^{H-H}\left(Q^{2}\right), J^{H-H}\left(Q^{2}\right), D^{H-H}\left(Q^{2}\right)$, and $\quad \bar{c}^{H-H}\left(Q^{2}\right) \quad$ and; $\quad F^{H-H}(0)=A^{H-H}\left(Q^{2}=0\right)$, $J^{H-H}\left(Q^{2}=0\right), D^{H-H}\left(Q^{2}=0\right)$, and $\bar{c}^{H-H}\left(Q^{2}=0\right)$. We should say here that the light-cone QCD sum rule is reliable only at $Q^{2} \geq 1.0 \mathrm{GeV}^{2}$. Our numerical values for the form factors at $Q^{2}=0$, obtained using the shape parameters from the QCD sum rules, are presented in Table II.

To our best knowledge, this is the first study in the literature dedicated to the investigation of the hyperon's gravitational form factors. Therefore, experimental data or theoretical estimations are not yet available to compare

TABLE II. The values of form factors at $Q^{2}=0$ obtained using the multipole fit parametrization and the values of shape parameters from QCD sum rules.

\begin{tabular}{lccc}
\hline \hline Transition & $F^{H-H}(0)$ & $m_{p}\left(\mathrm{GeV}^{-2}\right)$ & $p$ \\
\hline$\Sigma-\Sigma$ & $A(0)=0.86 \pm 0.12$ & $1.15 \pm 0.08$ & $3.2 \pm 0.2$ \\
& $J(0)=0.40 \pm 0.06$ & $1.16 \pm 0.10$ & $3.2 \pm 0.2$ \\
& $D(0)=-2.65 \pm 0.25$ & $1.13 \pm 0.07$ & $3.2 \pm 0.1$ \\
$\Xi-\Xi$ & $\bar{c}(0)=-$ & - & - \\
& $A(0)=0.53 \pm 0.05$ & $1.10 \pm 0.08$ & $3.2 \pm 0.2$ \\
& $J(0)=0.25 \pm 0.02$ & $0.93 \pm 0.06$ & $3.2 \pm 0.2$ \\
& $D(0)=-2.30 \pm 0.18$ & $1.02 \pm 0.06$ & $3.2 \pm 0.1$ \\
& $\bar{c}(0)=-0.12 \pm 0.01$ & $0.98 \pm 0.06$ & $3.8 \pm 0.2$ \\
$\Lambda-\Lambda$ & $A(0)=0.58 \pm 0.10$ & $1.10 \pm 0.10$ & $3.2 \pm 0.2$ \\
& $J(0)=0.32 \pm 0.06$ & $1.20 \pm 0.10$ & $3.2 \pm 0.2$ \\
& $D(0)=-2.53 \pm 0.12$ & $1.10 \pm 0.05$ & $3.2 \pm 0.1$ \\
& $\bar{c}(0)=-0.10 \pm 0.01$ & $1.10 \pm 0.10$ & $3.8 \pm 0.2$ \\
\hline \hline
\end{tabular}

them with our numerical results. However, we may compare these results with the nucleon's gravitational form factors especially for the form factor $D^{H-H}\left(Q^{2}\right)$. Various estimations for the $D^{N-N}(0)$ are as follows. Lattice QCD predictions: $D^{N-N}(0)=-1.76 \pm 0.09$ [30], $D^{N-N}(0)=$ $-2.27 \pm 0.30$ [31], chiral perturbation theory estimates: $D^{N-N}(0)=-1.93 \pm 0.06$ [10], light-cone QCD sum rule results: $\quad D^{N-N}(0)=-2.63 \pm 0.22 \quad[35], \quad D^{N-N}(0)=$ $-2.29 \pm 0.58$ (set-I) [36], $D^{N-N}(0)=-2.05 \pm 0.40$ (setII) [36], KM15 global fit prediction: $D^{N-N}(0)=-2.18 \pm$ 0.21 [70] and JLab data: $D^{N-N}(0)=-2.11 \pm 0.46$ [71]. It should be noted here that the results of other theoretical approaches have been obtained at different renormalization scale and these results have been shifted to the renormalization scale $\mu^{2}=1 \mathrm{GeV}^{2}$ (For details, see e.g., [36]). As one can see from these predictions, the numerical results for the D-term of hyperons obtained in the present work are close to the nucleon's D-term and we see a reasonable SU(3) flavor violation.

As we previously mentioned, the shape parameters of DAs of hyperons are now available from lattice QCD [66], as well. Using these values by changing the scale of the parameters to the one used in the present study, we obtain the values of the form factors at $Q^{2}=0$ as presented in Table III. Comparing the values of the form factors in this table with those in Table II, we see that the central values differ from each other by $8 \%-34 \%$. These amounts of the changes are reasonable as the DAs of the baryons generally have strong dependencies to the shape parameters.

After obtaining the gravitational form factors, we can use them to calculate some mechanical properties associated with the hyperons such as mechanical radius square $\left(\left\langle r_{\text {mech }}^{2}\right\rangle\right)$ as well as energy $(\mathcal{E})$ and pressure $\left(p_{0}\right)$ distributions at the center of these particles. The related formulas are given as [2],

TABLE III. The values of form factors at $Q^{2}=0$ obtained using the multipole fit parametrization and the values of shape parameters from lattice QCD.

\begin{tabular}{|c|c|c|c|}
\hline Transition & $F^{H-H}(0)$ & $m_{p}\left(\mathrm{GeV}^{-2}\right)$ & $p$ \\
\hline$\Sigma-\Sigma$ & $\begin{aligned} A(0) & =0.98 \pm 0.18 \\
J(0) & =0.44 \pm 0.05 \\
D(0) & =-2.90 \pm 0.40 \\
\bar{c}(0) & =-\end{aligned}$ & $\begin{aligned} & 1.13 \pm 0.08 \\
& 1.09 \pm 0.09 \\
& 1.09 \pm 0.07 \\
&-\end{aligned}$ & $\begin{array}{l}3.2 \pm 0.2 \\
3.2 \pm 0.2 \\
3.2 \pm 0.1 \\
\quad-\end{array}$ \\
\hline$\Xi-\Xi$ & $\begin{array}{c}A(0)=0.70 \pm 0.07 \\
J(0)=0.22 \pm 0.04 \\
D(0)=-2.85 \pm 0.30 \\
\bar{c}(0)=-0.13 \pm 0.02\end{array}$ & $\begin{array}{r}1.16 \pm 0.08 \\
0.93 \pm 0.05 \\
1.0 \pm 0.05 \\
0.95 \pm 0.07\end{array}$ & $\begin{array}{l}3.2 \pm 0.2 \\
3.2 \pm 0.2 \\
3.2 \pm 0.1 \\
3.8 \pm 0.2\end{array}$ \\
\hline$\Lambda-\Lambda$ & $\begin{aligned} A(0) & =0.87 \pm 0.17 \\
J(0) & =0.42 \pm 0.06 \\
D(0) & =-2.70 \pm 0.50 \\
\bar{c}(0) & =-0.11 \pm 0.01\end{aligned}$ & $\begin{array}{l}1.22 \pm 0.10 \\
1.20 \pm 0.17 \\
1.15 \pm 0.10 \\
1.13 \pm 0.11\end{array}$ & $\begin{array}{l}3.2 \pm 0.2 \\
3.2 \pm 0.2 \\
3.2 \pm 0.1 \\
3.8 \pm 0.2\end{array}$ \\
\hline
\end{tabular}


TABLE IV. The values of mechanical quantities for hyperons using the shape parameters from QCD sum rules.

\begin{tabular}{lccc}
\hline \hline Transition & $p_{0}\left(\mathrm{GeV} / \mathrm{fm}^{3}\right)$ & $\mathcal{E}\left(\mathrm{GeV} / \mathrm{fm}^{3}\right)$ & $\left\langle r_{\text {mech }}^{2}\right\rangle\left(\mathrm{fm}^{2}\right)$ \\
\hline$\Sigma-\Sigma$ & $0.54 \pm 0.14$ & $2.41 \pm 0.63$ & $0.61 \pm 0.06$ \\
$\Xi-\Xi$ & $0.62 \pm 0.10$ & $2.09 \pm 0.62$ & $0.52 \pm 0.04$ \\
$\Lambda-\Lambda$ & $0.59 \pm 0.15$ & $2.08 \pm 0.56$ & $0.59 \pm 0.05$ \\
\hline \hline
\end{tabular}

TABLE V. The values of mechanical quantities for hyperons using the shape parameters from lattice QCD.

\begin{tabular}{lccc}
\hline \hline Transition & $p_{0}\left(\mathrm{GeV} / \mathrm{fm}^{3}\right)$ & $\mathcal{E}\left(\mathrm{GeV} / \mathrm{fm}^{3}\right)$ & $\left\langle r_{\text {mech }}^{2}\right\rangle\left(\mathrm{fm}^{2}\right)$ \\
\hline$\Sigma-\Sigma$ & $0.64 \pm 0.14$ & $2.86 \pm 0.68$ & $0.58 \pm 0.06$ \\
$\Xi-\Xi$ & $0.69 \pm 0.10$ & $2.45 \pm 0.60$ & $0.54 \pm 0.04$ \\
$\Lambda-\Lambda$ & $0.56 \pm 0.13$ & $2.23 \pm 0.51$ & $0.62 \pm 0.08$ \\
\hline \hline
\end{tabular}

$$
\begin{gathered}
p_{0}=-\frac{1}{24 \pi^{2} m_{H}} \int_{0}^{\infty} d y y \sqrt{y}[D(y)-\bar{c}(y)], \\
\mathcal{E}=\frac{m_{H}}{4 \pi^{2}} \int_{0}^{\infty} d y \sqrt{y}\left[A(y)+\frac{y}{4 m_{H}^{2}}[A(y)-2 J(y)\right. \\
+D(y)+\bar{c}(y)]], \\
\left\langle r_{\text {mech }}^{2}\right\rangle=\frac{6 D(0)}{\int_{0}^{\infty} d y D(y)},
\end{gathered}
$$

where $y=Q^{2}$.

The numerical results of mechanical properties of hyperons are presented in Tables IV and V, for the shape parameters from the QCD sum rules and lattice QCD, respectively. Comparing the central values of the parameters from these two tables, we see that the differences among the corresponding values lie in the interval $4 \%-16 \%$. The minimum difference belongs to the $\left\langle r_{\text {mech }}^{2}\right\rangle_{\Xi}$ and the maximum difference between the values is related to the $\mathcal{E}_{\Sigma}$. Our results may be checked via different theoretical approaches.

\section{SUMMARY AND CONCLUDING REMARKS}

We calculated the quark part of the gravitational form factors of hyperons by using the light-cone QCD sum rule method. We used the general forms of the interpolating currents for the hyperons and the quark part of the energymomentum current together with the DAs of the hyperonic states calculated via both the QCD sum rules and lattice QCD. These form factors carry information about the angular momentum and mass as well as energy and pressure distributions inside the baryons. We obtained that the $Q^{2}$ dependence of the hyperon's gravitational form factors are nicely characterized by a multipole fit function. Using these fit functions, we obtained the static form factors, i. e., their values at $Q^{2}=0$. The hyperons under study are unstable and have a small lifetime, hence measurements on their properties are very difficult compared with the nucleons. We have a good experimental information on the electromagnetic form factors of nucleons, even for the D-term of the nucleons we have some data provided by JLab. We also have a good knowledge of theory on the electromagnetic, axial, tensor, and gravitational form factors of the nucleons. However, the number of works dedicated to the electromagnetic, axial, and tensor form factors of hyperons are very limited. In the case of gravitational form factors there are no theory predictions are available. Hence, our results may be checked via different methods in future. We hope we can have experimental data on the gravitational form factors of hyperons in future. Comparison of different results on these form factors can help us gain valuable information on the internal structures of hyperons. Our analyses show that using the shape parameters from the QCD sum rules or lattice QCD changes the results of form factors at $Q^{2}=0$ maximally by $34 \%$.

As a by-product, we calculated some mechanical properties of hyperons such as the mean mechanical radius square as well as the pressure and energy distributions at the center of these particles, as well. The results indicate that the $\left\langle r_{\text {mech }}^{2}\right\rangle_{H}$ for $H=\Sigma, \Lambda$ are considerably bigger than that of the nucleons, $\left\langle r_{\text {mech }}^{2}\right\rangle_{N}=(0.52-0.54) \pm 0.05 \mathrm{fm}^{2}$, obtained via the same method in Ref. [36]. However, the mean radius square for $\Xi$ hyperon is comparable with that of the nucleon. We observed that switching the values of the shape parameters from QCD sum rules to those from lattice QCD, changes the results of the mechanical parameters by $4 \%-16 \%$. Our results on the mechanical properties of hyperons may be checked via different theoretical approaches as well as Lattice QCD. Any future experimental data and their comparison with our estimations can give valuable knowledge on the internal structure and geometrical shapes of hyperons.

\section{ACKNOWLEDGMENTS}

The work of U. Ö. is supported under 2218-National Postdoctoral Research Scholarship Programme by the scientific and technological research council of Turkey (TUBITAK). 
[1] H. Pagels, Energy-momentum structure form factors of particles, Phys. Rev. 144, 1250 (1966).

[2] M. V. Polyakov and P. Schweitzer, Forces inside hadrons: Pressure, surface tension, mechanical radius, and all that, Int. J. Mod. Phys. A 33, 1830025 (2018).

[3] M. V. Polyakov, Generalized parton distributions and strong forces inside nucleons and nuclei, Phys. Lett. B 555, 57 (2003).

[4] I. Kobzarev and L. Okun, Gravitational interaction of fermions, Zh. Eksp. Teor. Fiz. 43, 1904 (1962).

[5] J.-W. Chen and X.-D. Ji, Leading Chiral Contributions to the Spin Structure of the Proton, Phys. Rev. Lett. 88, 052003 (2002).

[6] A. V. Belitsky and X. Ji, Chiral structure of nucleon gravitational form-factors, Phys. Lett. B 538, 289 (2002).

[7] S.-i. Ando, J.-W. Chen, and C.-W. Kao, Leading chiral corrections to the nucleon generalized parton distributions, Phys. Rev. D 74, 094013 (2006).

[8] M. Diehl, A. Manashov, and A. Schafer, Chiral perturbation theory for nucleon generalized parton distributions, Eur. Phys. J. A 29, 315 (2006).

[9] M. Diehl, A. Manashov, and A. Schafer, Generalized parton distributions for the nucleon in chiral perturbation theory, Eur. Phys. J. A 31, 335 (2007).

[10] M. Dorati, T. A. Gail, and T. R. Hemmert, Chiral perturbation theory and the first moments of the generalized parton distributions in a nucleon, Nucl. Phys. A798, 96 (2008).

[11] M. J. Neubelt, A. Sampino, J. Hudson, K. Tezgin, and P. Schweitzer, Energy momentum tensor and the $D$-term in the bag model, arXiv:1911.08906.

[12] M. V. Polyakov and H.-D. Son, Nucleon gravitational form factors from instantons: Forces between quark and gluon subsystems, J. High Energy Phys. 09 (2018) 156.

[13] V. Yu. Petrov, P. V. Pobylitsa, M. V. Polyakov, I. Bornig, K. Goeke, and C. Weiss, Off-forward quark distributions of the nucleon in the large N(c) limit, Phys. Rev. D 57, 4325 (1998).

[14] P. Schweitzer, S. Boffi, and M. Radici, Polynomiality of unpolarized off forward distribution functions and the D term in the chiral quark soliton model, Phys. Rev. D 66, 114004 (2002).

[15] J. Ossmann, M. V. Polyakov, P. Schweitzer, D. Urbano, and $\mathrm{K}$. Goeke, The generalized parton distribution function $\left(E^{u}+E^{d}\right)(x, \xi, t)$ of the nucleon in the chiral quark soliton model, Phys. Rev. D 71, 034011 (2005).

[16] M. Wakamatsu and H. Tsujimoto, The Generalized parton distribution functions and the nucleon spin sum rules in the chiral quark soliton model, Phys. Rev. D 71, 074001 (2005).

[17] M. Wakamatsu and Y. Nakakoji, Generalized form factors, generalized parton distributions and the spin contents of the nucleon, Phys. Rev. D 74, 054006 (2006).

[18] K. Goeke, J. Grabis, J. Ossmann, P. Schweitzer, A. Silva, and D. Urbano, The pion mass dependence of the nucleon form-factors of the energy momentum tensor in the chiral quark-soliton model, Phys. Rev. C 75, 055207 (2007).

[19] K. Goeke, J. Grabis, J. Ossmann, M. V. Polyakov, P. Schweitzer, A. Silva, and D. Urbano, Nucleon form-factors of the energy momentum tensor in the chiral quark-soliton model, Phys. Rev. D 75, 094021 (2007).
[20] J.-H. Jung, U. Yakhshiev, and H.-C. Kim, Energymomentum tensor form factors of the nucleon within asoliton model, J. Phys. G 41, 055107 (2014).

[21] J.-H. Jung, U. Yakhshiev, H.-C. Kim, and P. Schweitzer, Inmedium modified energy-momentum tensor form factors of the nucleon within the framework of a $\pi-\rho-\omega$ soliton model, Phys. Rev. D 89, 114021 (2014).

[22] J.-H. Jung, U. Yakhshiev, and H.-C. Kim, Modification of generalized vector form factors and transverse charge densities of the nucleon in nuclear matter, Phys. Rev. D 93, 054016 (2016).

[23] M. Wakamatsu, On the D-term of the nucleon generalized parton distributions, Phys. Lett. B 648, 181 (2007).

[24] B. Pasquini, M. V. Polyakov, and M. Vanderhaeghen, Dispersive evaluation of the D-term form factor in deeply virtual Compton scattering, Phys. Lett. B 739, 133 (2014).

[25] C. Cebulla, K. Goeke, J. Ossmann, and P. Schweitzer, The Nucleon form-factors of the energy momentum tensor in the Skyrme model, Nucl. Phys. A794, 87 (2007).

[26] H.-C. Kim, P. Schweitzer, and U. Yakhshiev, Energymomentum tensor form factors of the nucleon in nuclear matter, Phys. Lett. B 718, 625 (2012).

[27] P. Hagler, J. W. Negele, D. B. Renner, W. Schroers, T. Lippert, and K. Schilling, Moments of nucleon generalized parton distributions in lattice QCD, Phys. Rev. D 68, 034505 (2003).

[28] N. Mathur, S. J. Dong, K. F. Liu, L. Mankiewicz, and N. C. Mukhopadhyay, Quark orbital angular momentum from lattice QCD, Phys. Rev. D 62, 114504 (2000).

[29] M. Gockeler, R. Horsley, D. Pleiter, P. E. L. Rakow, A. Schafer, G. Schierholz, and W. Schroers, Generalized Parton Distributions from Lattice QCD, Phys. Rev. Lett. 92, 042002 (2004).

[30] J. D. Bratt et al., Nucleon structure from mixed action calculations using $2+1$ flavors of asqtad sea and domain wall valence fermions, Phys. Rev. D 82, 094502 (2010).

[31] P. Hagler et al., Nucleon generalized parton distributions from full lattice QCD, Phys. Rev. D 77, 094502 (2008).

[32] D. Brommel et al., Moments of generalized parton distributions and quark angular momentum of the nucleon, Proc. Sci., LATTICE2007 (2007) 158.

[33] J. W. Negele et al., Insight into nucleon structure from lattice calculations of moments of parton and generalized parton distributions, Nucl. Phys. B, Proc. Suppl. 128, 170 (2004).

[34] M. Deka et al., Lattice study of quark and glue momenta and angular momenta in the nucleon, Phys. Rev. D 91, 014505 (2015).

[35] I. V. Anikin, Gravitational form factors within light-cone sum rules at leading order, Phys. Rev. D 99, 094026 (2019).

[36] K. Azizi and U. Özdem, Nucleon's energy-momentum tensor form factors in light-cone QCD, Eur. Phys. J. C 80, 104 (2020).

[37] C. Lorcé, H. Moutarde, and A. P. Trawiński, Revisiting the mechanical properties of the nucleon, Eur. Phys. J. C 79, 89 (2019).

[38] U. Özdem and K. Azizi, Gravitational transition form factors of $N(1535) \rightarrow N$, Phys. Rev. D 101, 054031 (2020). 
[39] V. M. Braun and I. E. Filyanov, QCD sum rules in exclusive kinematics and pion wave function, Yad. Fiz. 50, 818 (1989) [Z. Phys. C 44, 157 (1989)].

[40] I. I. Balitsky, V. M. Braun, and A. V. Kolesnichenko, Radiative decay $\sigma^{+} \rightarrow \mathrm{p} \gamma$ in quantum chromodynamics, Nucl. Phys. B312, 509 (1989).

[41] V.L. Chernyak and I. R. Zhitnitsky, $B$ meson exclusive decays into baryons, Nucl. Phys. B345, 137 (1990).

[42] M. E. Carrillo-Serrano, W. Bentz, A. W. Cloët, and I. C. Thomas, Baryon octet electromagnetic form factors in a confining NJL model, Phys. Lett. B 759, 178 (2016).

[43] X. Y. Liu, K. Khosonthongkee, A. Limphirat, and Y. Yan, Study of baryon octet electromagnetic form factors in perturbative chiral quark model, J. Phys. G 41, 055008 (2014).

[44] F.-J. Jiang and B.C. Tiburzi, Hyperon electromagnetic properties in two-flavor chiral perturbation theory, Phys. Rev. D 81, 034017 (2010).

[45] H.-W. Lin and K. Orginos, Strange baryon electromagnetic form factors and SU(3) flavor symmetry breaking, Phys. Rev. D 79, 074507 (2009).

[46] G. Ramalho and K. Tsushima, Octet baryon electromagnetic form factors in a relativistic quark model, Phys. Rev. D 84, 054014 (2011).

[47] T. M. Aliev, Y. Oktem, and M. Savci, Electromagnetic transitions among octet and decuplet baryons in QCD, Phys. Rev. D 88, 036002 (2013).

[48] T. M. Aliev, A. Ozpineci, and M. Savci, Octet baryon magnetic moments in light cone QCD sum rules, Phys. Rev. D 66, 016002 (2002); Erratum, Phys. Rev. D 67, 039901 (2003).

[49] T. M. Aliev, K. Azizi, and M. Savci, Electric quadrupole and magnetic octupole moments of the light decuplet baryons within light cone QCD sum rules, Phys. Lett. B 681, 240 (2009).

[50] T. M. Aliev, K. Azizi, and M. Savci, Electromagnetic form factors of octet baryons in QCD, Phys. Lett. B 723, 145 (2013).

[51] T. M. Aliev, K. Azizi, and M. Savci, Analysis of $\gamma^{*} \Lambda \rightarrow \Sigma^{0}$ transition in QCD, Phys. Rev. D 87, 096013 (2013).

[52] T. M. Aliev and A. Ozpineci, Magnetic moments of decuplet baryons in light cone QCD, Phys. Rev. D 62, 053012 (2000).

[53] H.-W. Lin and K. Orginos, First calculation of hyperon axial couplings from lattice QCD, Phys. Rev. D 79, 034507 (2009).

[54] G. Ramalho and K. Tsushima, Axial form factors of the octet baryons in a covariant quark model, Phys. Rev. D 94, 014001 (2016).
[55] T. Ledwig, J. Martin Camalich, L. S. Geng, and M. J. Vicente Vacas, Octet-baryon axial-vector charges and SU(3)-breaking effects in the semileptonic hyperon decays, Phys. Rev. D 90, 054502 (2014).

[56] A. Kucukarslan, U. Ozdem, and A. Ozpineci, Isovector axial vector form factors of octet-decuplet hyperon transition in QCD, J. Phys. G 42, 015001 (2015).

[57] G. Erkol and A. Ozpineci, Isovector axial-vector form factors of octet baryons in QCD, Phys. Rev. D 83, 114022 (2011).

[58] K.-S. Choi, W. Plessas, and R. F. Wagenbrunn, Axial charges of octet and decuplet baryons, Phys. Rev. D 82, 014007 (2010).

[59] G. Erkol, M. Oka, and T. T. Takahashi, Axial charges of octet baryons in two-flavor lattice QCD, Phys. Lett. B 686, 36 (2010).

[60] F.-J. Jiang and B. C. Tiburzi, Hyperon axial charges in twoflavor chiral perturbation theory, Phys. Rev. D 80, 077501 (2009).

[61] F.-J. Jiang and B. C. Tiburzi, Chiral corrections to hyperon axial form factors, Phys. Rev. D 77, 094506 (2008).

[62] T. Ledwig, A. Silva, and H.-C. Kim, Tensor charges and form factors of $\mathrm{SU}(3)$ baryons in the self-consistent SU(3) chiral quark-soliton model, Phys. Rev. D 82, 034022 (2010).

[63] A. kucukarslan, U. Ozdem, and A. Ozpineci, Tensor form factors of the octet hyperons in QCD, Phys. Rev. D 94, 094010 (2016).

[64] Y.-L. Liu and M.-Q. Huang, Distribution amplitudes of $\Sigma$ and $\Lambda$ and their electromagnetic form factors, Nucl. Phys. A821, 80 (2009).

[65] Y.-L. Liu and M.-Q. Huang, Light-cone distribution amplitudes of $\Xi$ and their applications, Phys. Rev. D 80, 055015 (2009).

[66] G. S. Bali et al., Light-cone distribution amplitudes of octet baryons from lattice QCD, Eur. Phys. J. A 55, 116 (2019).

[67] P. Wein and A. Schäfer, Model-independent calculation of $\mathrm{SU}(3)_{f}$ violation in baryon octet light-cone distribution amplitudes, J. High Energy Phys. 05 (2015) 073.

[68] V. M. Braun, A. Lenz, and M. Wittmann, Nucleon form factors in QCD, Phys. Rev. D 73, 094019 (2006).

[69] M. Tanabashi et al., Review of particle physics, Phys. Rev. D 98, 030001 (2018).

[70] I. V. Anikin et al., Nucleon and nuclear structure through dilepton production, Acta Phys. Pol. B 49, 741 (2018).

[71] V. D. Burkert, L. Elouadrhiri, and F. X. Girod, The pressure distribution inside the proton, Nature (London) 557, 396 (2018). 